\title{
Reumatología y tuberculosis. ¿Se debería considerar estudios controlados en nuestro país?
}

\author{
Rheumatology and tuberculosis. Should we consider controlled studies in our country?
}

\section{Sr. Editor:}

La incidencia de cuadros reumatológicos ha presentado un incremento proporcional con el aumento de la esperanza de vida a nivel mundial, esto ha devenido en un impacto en la calidad de vida, productividad de la población económicamente activa y en los costes sanitarios. Debido a esto la Organización Mundial de la Salud (OMS) declaró el decenio de 2000 - 2010 como la década de los huesos y las articulaciones, considerando que los cuadros reumatológicos se ubicarían en la cuarta causa de discapacidad para el 2020 (1).

A la fecha se han logrado avances en el control de la enfermedad mediante terapias moduladoras (antirreumáticos modificadores de la enfermedad, terapia biológica); sin embargo, tal manipulación del sistema inmune ha traído un incremento en el riesgo de contraer infecciones oportunistas, entre ellas (dado nuestro contexto) la tuberculosis.

Es conocido que existe una asociación entre las enfermedades reumatológicas y la tuberculosis, siendo especialmente significativa en artritis reumatoide (OR 2,0 p <0,001), lupus eritematoso sistémico (OR $4,90 \mathrm{p}<0.001)$ y Sjögren (OR 6,11 p<0,001) (2). Esto podría ser atribuido a la fisiopatología de los cuadros autoinmunes (asplenia funcional, disminución del complemento sérico, disminución de las células $\mathrm{T}$ citotoxicas, alteración de las células T supresoras, etc.) (3), así como también al tratamiento farmacológico inmunosupresor que aumentarían el riesgo a infecciones por patógenos intracelulares, como las micobacterias.
The American Thorax Society considera la profilaxis con isoniazida por nueves meses cuando la prueba de tuberculina es mayor a $10 \mathrm{~mm}$ en pacientes con enfermedades que aumente el riesgo de contraer TBC (como las reumatológicas) y cuando sea mayor a $5 \mathrm{~mm}$ en todos los que reciban más de $15 \mathrm{mg}$ de prednisona o equivalente al día. Además, se recomienda la prueba de tuberculina anual a los que presenten los factores de riesgo antes descritos (4). Esto fue documentado en India, donde se evidenció que la profilaxis con isoniacida en pacientes con lupus logró reducir la incidencia de tuberculosis de 11 a $2 \%$ (3), disminuyendo la mortalidad asociada a esta infección, así como los costes sanitarios.

Actualmente no se cuenta con estudios prospectivos controlados en nuestro medio donde se pueda evidenciar la tasa de incidencia de tuberculosis en pacientes con cuadros reumatológicos, especialmente en lupus, pese a que en nuestro país la tuberculosis es un problema de salud pública. Del mismo modo se desconoce si la profilaxis con isoniazida en estos pacientes ha logrado tener el impacto esperado.

A manera de conclusión, es importante la ejecución de estudios epidemiológicos que permitan establecer la incidencia de tuberculosis en pacientes con cuadros reumatológicos y la repercusión en la evolución de la enfermedad, del mismo modo estos estudios darían la oportunidad de realizar ensayos controlados para evidenciar si el impacto de la profilaxis con isoniazida cumpliría con el objetivo planteado, dado el polimorfismo genético de nuestra población.

Ernesto Zavala-Flores ${ }^{1, a}$ 


\section{Correspondencia:}

Ernesto Moises Zavala Flores

Av. Próceres de la independencia SJL, Lima, Perú.

Teléfono: 51944270757

Correo electrónico: ernestozav123@gmail.com

\section{REFERENCIAS BIBLIOGRÁFICAS}

1. WoolfAD, Pfleger B. Burden of major musculoskeletal conditions. Bull World Health Organ. 2003; 81(9):646-56.

2. Lu MC, Lai C, Tsai C, Koo M, Lai N. Increased risk of pulmonary and extra-pulmonary tuberculosis in patients with rheumatic diseases. Int J Tuberc Lung Dis. 2015; 19(12):1500-6.
3. Arenas M, Hidalgo C, Jimenez J. Tuberculosis in patients with systemic lupus erythematosus: Spain's situation, Reumatol Clin. 2013; 9(6):369-72.

4. Targeted tuberculin testing and treatment of latent tuberculosis infection. This official statement of the American Thoracic Society was adopted by the ATS Board of Directors, July 1999. This is a Joint Statement of the American Thoracic Society (ATS) and the Centers for Disease Control and Prevention (CDC). This statement was endorsed by the Council of the Infectious Diseases Society of America. (IDSA), September 1999, and the sections of this statement. Am J Respir Crit Care Med. 2000; 161(4 Pt 2):S22147.

Recibido: 12/07/2017 